\title{
Urocitograma y parto (futuro en la obstetricia)
}

\author{
Doctores G. López-Escobar (\%) y César Mendoza (*)
}

\section{INFORME PRELIMINAR}

La determinación de la fecha probable del parto se ha basado desde hace largo tiempo en la medida de la altura uterina por uno u otro método, la estimación del peso fetal, la correlación de la fecha de la última menstruación y de los primeros movimientos percibidos por la paciente, la madurez del cuello y en ocasiones en estudios radiológicos. Pero como de todes ıes bien sabido esta apreciación sometida a grandes causas de error es tan solo muy aproximada. Así mismo aunque es corriente hablar de senilidad placentaria a los nueve meses, puesto que se piensa que la placenta es un órgano que sufre un proceso de envejecimiento acelerado, las investigaciones hormonales no han servido para fijar la fecha del parto ya que no. hay acuerdo entre los investigadores, pues a tiempo que unos iseñalan fibrosis, endarteritis a infartos (Grosser, Young, Bartholomew, Eden, Frases, Williams) (11.) y disminución progresiva del consumo de oxígeno (Wang, Hellman) (1.1), otros anotan que la cantidad de estrógeno y progesterona. producida por la placenta alcanza sus mayores niveles a término (Smith y Smith, Cohe, Probstner) (1.1) y que la taisa de transfarencia del sodio radioactivo aumenta seis veces de le $12^{a}$ o la $40^{a}$ semana (Gellhorn y Flexner) (111) lo que indicaría una mayor actividad placentaria a término. Por esto la investigación sobre la fecha del parto. se ha dirigido hacia un nuevo campo: la citologia.

Desde 19:25 Papanicolaou (16) morstró las diferencias entre el frotis vaginal de una mujer embarazada y una no gestante y sus descripcione's de las "Células Naviculares" y de las llamadas "Cê-

(*) Profesor Agregado de Ginecología de la Universidad Nacional FACS FICS.

(**) Jefe de Laboratorios. Instituıo Nacicnal de Ginecología, Bogotá. 
lulas Post-Partum" han permanecido como clásicas. Poster ‘ormente se han publicado numerosos trabajos sobre la citologia de la gestante, orientados unos hacia el diagnóstico del embarazo, otros al descubrimiento precoz de un aborto inminente o un parto prematuro. o a los cambios que se suceden en el último trimestre $y$ en el pre-parto inmediato. Pundell y van Meensel (9-10) describieron el aumento del índice eosinofílico y picnótico cor aparición de elementos superficiales como indicadores de un parto próximo. $Y$ la magnifica demostración de Lemberg Sigfried, stamm y de Wateville (1-3) en el congreso de Ginebra en 1954 hizo hincapiể sobre una correlación de un noventa por ciento entro" los cambios citológicos vaginales "a término" y la fecha de confinamiento, estableciendo que las modificaciones del frotis permiten escoger mejor los casos susceptibles de ser inducidos con exito. Mas luego Barnes y Zuspan (5) en 1956 solo comprobaron un "setenta y tres por ciento de certeza en el criterio citológico propuesto para la determinación de la fecha del parto".

Por otra parte bien sabido es, en especial gracias a los trabajos de la escuela argentina (Del Castillo, Argonz, Galli Mainini, Pérez Gambet, etc.), (12-14) qui existe un paralelismo entre las modificaciones citológicas del sedimento urinario y las de la citología vaginal. Así, ya en 1950 McGallin, Taylor y Whitehead (4) hallaron que el sedimento urinario presenta una citolog a típica durante el embarazo y que la semana que precede al parto hay alteraciones que consisten en "Descamación acentuada y presencia de células con citoplasma intensamente teñido y núcleo vesicular grande". Ultimamente los trabajos de los mexicanos Alvarez Bravo y González Ramos (6-7-8) sobre el urocitograma al final del embarazo han puntualizado y reafirmado estas investigaciones, pues en su experiencia: "Es posible determinar la proximidad del parto con una antelación de uno a siete días en el ciento por ciento de los casos". Aunque técnicamente es un poco más difícil, las ventajas del urocitograma sobre la citologia vaginal en el estudio seriado que es necesario hacer en una gestante para la determinación de la fecha deI parto, estriban en la facilidad de la toma de la muestra, sin maniobras intravaginales, sin que la paciente acuda al laboratorio y sin la contaminación con flujos vaginales de una $u$ otra índole.

Pero ¿cuál es el propósito de predecir la ficha del parto? Existen grupos de condiciones que se beneficiarian de esta determinación. Así, el tan debatido sindrome post-madurez; la fija- 
ción de la fecha de una cesárea electiva de repetición; la escogencia dé la fecha de parto en una gestante cuyo embarazo deba sur interrumpido antes del término por una complicación dịabética o de otro tipo, etc. Ella nos ayudaría tambiéh a prever y tratar abortos o partos prematuros inminentes, scbre todo en aquellas pacientes que hacen abortos o partos prematuros de repetición y in el diagnóstico de la muerte fetal in útero. Finalmente estamos en todo de acuerdo con Alvarez Bravo (7) en que: en el futuro de la obstetricia está la inducción electiva del parto considerada "como el desencadenamiento de un parto inminente". Alvarez (7) hace suyas las palabras de Hanley (15) cuando pregunta: "¿Aunque el parto tenga en sí mismo el concepto de ser una emergencia es indebido el tratar de veneer las inadecuadas condiciones inherentes a la emergencia cuando vemos el beneficio que proporciona este esfuerzo en otros campos de la medicina? ¿Debemos continuar permaneciendo como comadronas expectantes o actuar como médicos con adecuada preparación toco-quirúrgica capaces de mejorar sus elementos de trabajo y de precisar las mejores condiciones del desarrollo del parto?" $Y$ cen nuestro medio en las que múltiples dificultades de transporte, etc. acentúan las distancias, esta inducción electiva considerada como el "desencadienamiento de un parto inmininte" ¿no obviaria muchas carreras y afanes desventajosos para la gestante?

Es obvio que el urocitograma sería tan solo un medio coadyuvante en la determinación de la fecha del parto, pues para la fijación de ésta deben convergir y concordar todos los datos del examen tanto clínico como de laboratorio.

Queremos, además, dejar bien en claro que presentamos este tema únicamente a mera información y de reporte preliminar, ilusionados con los trabajos de Alvarez y González (6-7-8), pues a nesar de que hemos venido trabajando al respecto desde marzo del presente año, nuestro criterio. sobre el urocitograma como medio cierto de indicación de un parto inminenté aún no está bien formado; criterio que no está tampoco bien unificado entre los distintos autores, ya que si analizamos los estudios citológicos realizados durante el embarazo, ya sea de secreciones vaginales o sedimento urinario, encontramos acuerdo en cuanto se refiere a los hallazgos durante el tercer trimestre, y a la existencia de modificaciones en los días que preceden ál parto, pero estas últimas son valoradas en forma diferente por.. los distintos autores. 
Van Meensel (9-10) anota un ligero aumento de IE e IP, cuyos límites superiores en el tercer trimestre fija en 6 y $20 \%$, respectivamente; aumento de las células superficiales y de escamación en placas.

Lemborg Sigfried y Satumm (1-3) encuentran sin significado los cambios de IE e IP y describen los cuadros de:

a) Embarazo avanzado no a término.

b) Embarazo cerca del término.

c) Embarazo a término.

El primero se caracteriza por marcada descamación con predominio de células intermedias muchas de tipo navicular, buena vitalidad celular, escasos leucocitos y poco moco. Indica que el parto no se presentará antes de 10 días.

El segundo por disminución en la descamación, en las células intermedias y naviculares y en la vitalidad celular, con aumento de los leucocitos y el moco. Indica que el parto se presentará dentro de los diez días siguientes.

El tercero muestra acentuación en ios cambios anteriores con notorio aumento de células superficiales y la desvitalización celular que unida a la abundancia de leucocitos y moco da al frotis un aspecto sucio. Indica que el parto se presentará dentro de los dos dias siguientes.

McCallin, Taylor y Whitehead (4) en el sedimento urinario reportan la presencia de células naviculares más pequeñas a medida que el embarazo avanza hacia el noveno mes, aumento de la picnosis, vacuolización, granulación y eosinofilia en las células superficiales. En la semana que precede al parto encuentran aumento de la celularidad, agrupación de las células naviculares y superficiales y aparición de células semejantes a las descritas por Papanicolaou como die post-partum.

La aparente divergencia, entre los hallazgos de Lemberg Sigfried y McCallin y col, puede ser debida a que los estudios de los primeros se refieren a vagina y la de los segundos a sedimento urinario, en el cual una extensa descamación precede y acompaña la iniciación del parto, fenómeno que no se observa en el epitelio vaginal, aun cuando Davis y Hartman (citados por McCallin) (4) lo encuentran en los monos, en los cuales casi todo el epitelio vaginal descama antes de iniciarse el parto.

Alvarez Bravo y González Ramos (6-7-8) describen:

I) Cuadro de embarazo tardío con actividad esteroide normal, correspondiente a una gestación inmadura para el parto. Ca- 
racterizado por menos del $2 \%$ de células eosinófilas, menos del $10 \%$ de picnóticas, menos del $20 \%$ de superficiales y abundantes intermedias, naviculares y profundas.

II) Cuadros intermedios, interpretados como de comienzo de senilidad placentaria y caracterizados por IE del 2 al $10 \%$; IP hasta el $30 \%$ CS hasta el $40 \%$ con disminución de intermedias, naviculares y profundas.

III) Cuadro de madurez gravídica o de senilidad placentaria, caracterizado por IE de más del 10\%; IP de más del $30 \%$; CS de más del 40\% con ausencia o escasez de células naviculares, alteraciones nucleares y fenómenas de desvitalización celular. Llama la atención que buena parte de las células eosinófilas son de tipo intermedio.

El cuadro II se presenta entre 3 y 7 dias antes del parto y el III dentro de los 3 días anteriores.

Como se puede observar, los cambios citológicos y los períodos en que se presentan aunque tienen cierta inalogia no son completamente superponibles, lo cual en parte puede ser debido a diferencias en técnica, pero particularmente por la diversidad de los criterios empleados: forma de descamación, cambios nucleares, presencia de células atípicas, indices de e osinofilia y picnosis, cantidad de leucocitos y moco, etc.

\section{BIBLIOGRAFIA}

1. LEMBERG-SIGFRIED S. STAMM O.-Citologgie Vaginale a la fin de la Grossesse. Neuvelle Methode de Diagnostic de la Grossesse a terme: Exposition Scientifique Congres Int. de Ginec et Obst. Ginebra. 1954. Editado por Sandoz S. A. Basilea. págịna 62.

2. AEPPLI y HERMAN U.-Hormontherapie des Abortus Imminens. Exposition Scientifique. Congrés Int. de Gynec. et Obst. Ginebra. 1954. Editado por Sandoz, S. A, Besilea. Página 61.

3. LEMBERG-SIGFRIED S. STAMM O. y WATEVILLE H-Le Frottis Vaginal aux differents stades de la Crossesse Normale et Pathologique. La Presse Med. 63. No 76. noviembre 1955, página 1.558.

4. MCOALLIN P. E., TAYLOR E. S. y WHITEHEAD R. A. Study of the changes in the Cytology of the Urinary Sediment during the Menstrual Cycle and Pregnancy. Am, J. Obst. y Gynec. V. 60 No 1, july 1950, página 64.

5. BARNES A. C. y ZUSPAN E. P.-Vaginal Cytology as an Index of the Expected Date of Confinement. Am, J. Obst. y Gynec. V. 71. No 5. May. 1956. página 1080. 
6. ALVAREZ BRAVO A. y GONZALEZ RAMOS M.-El Urocitograma al final del embarazo. Gynec. y Obst. de México. V. XI. May. Jun. 1956, página 231.

7. ALVAREZ BRAVO A. y col. Vol.--Inducción Electiva del partc. Gynec. y Obst de México. y col. Vo1. V. XI. Mayo, Jun. 1956, páginis, 209.

8. ALVAREZ BRAVO y col,-La citología vaginal y la Citologia del. Sedimento urinario en el embarazo. Revista Colombiana de Obstetricia y Ginecología. V̆ol. VIII. No 2. Mar. Abr.. 1957, págin̊ 77.

9. VAN MEENSEL F.-Cylogie de la Gestation Normale. Sem des Hop. de París: Año 31, Ne $61 / 6$. Oct. 1955, página 273.

10. VAN MEENSEL F.-Cytologie de la Gestation Pathologique. Sem. des Hop. de París. An̂̀o 31. No 61/6. Oct. 1955, página 280.

11. BURNSTEIN R.-et al Histogenesis of Degenerative Precesses in the Normal Mature Placenta Am. J. Obsì y Gynee. Vol. 72. No 2. Aug/56, pág. 332.

12. PEREZ GAMBET C. y DEL CASTLLLO J.-EI Urocitogrema en Ginecología. Día Med. Vel. V. 28 (41) junio 1956, página 1.150.

13. ESCALLE G. y PELLTIER F.-Stude dẹs Frottị Vagiraux pendant les Suites des Couches. Gynec. et Obst. Vol. 49. No 5.1950.

14. DEL CASTILLO J. y col. Urocitograma: La Semana Médica. Vol..53. 1946, página 867 .

15. HANLEY B. J.-Amniotomy for the Elective Induction of Labor al or near Term. Western J. Of. Surg. Obst. 7 Gynec; Vol. 59. 1951, página 262 (Citado por Alvarez Bravo).

16. PAPANiCOLAOU G. N.-Proc. Scc. Exper. Biol. 7 Med. V. 22 1925, página 436.

17. PAPANICOLAOU G. N.-The Epithelia of Woman's Reproductive Organs. The Commonwealth. Fund Ed. New York. 1948.

18. LENCIONI J. L.-Comparative and Statistical Study of Vaginal and Urinary Sediment Smears. The $J$ of Clin End Metabolism. Vol. 13. No 3. Marzo $1^{\text {o }} 1953$, página 263 . 